\title{
Chapter 22 \\ Supporting Academic Integrity \\ in the Writing Centre: Perspectives \\ of Student Consultants
}

\author{
Kim Garwood
}

\begin{abstract}
Writing centres are often described as safe spaces where students can explore their ideas and concerns, including questions about how to use and cite sources without plagiarizing. In many Canadian writing centres, these issues are addressed by student consultants who provide effective and influential peer-to-peer support in individual consultations. Little research, however, has directly examined the perspectives of student consultants in providing academic integrity support. This chapter provides a synthesis of what literature currently exists on the role of student consultants in supporting academic integrity before describing a case study with student writing consultants at the University of Guelph. Using data gathered through a survey, this chapter examines the experience and perceptions of student consultants in providing academic integrity support. The findings suggest that academic integrity conversations often arise indirectly, through conversations about referencing or paraphrasing. Student writing consultants consistently position themselves as intermediaries, helping protect students from academic misconduct by using a range of directive and non-directive strategies.
\end{abstract}

Keywords Canada $\cdot$ Academic integrity $\cdot$ Plagiarism $\cdot$ Writing centres $\cdot$ Student consultants $\cdot$ Peer-to-peer support $\cdot$ University of Guelph

\footnotetext{
K. Garwood ( $\bowtie)$

University of Guelph, Guelph, Canada

e-mail: kgarwood@uoguelph.ca
}

S. E. Eaton and J. Christensen Hughes (eds.), Academic Integrity in Canada, 


\section{Introduction}

A great deal of academic integrity research in higher education has focused on students' perceptions, attitudes, and behaviours related to plagiarism, and much of this research has concluded that most students are baffled by academic integrity policies and fearful of inadvertently contravening them (Adam et al., 2017; Gullifer \& Tyson, 2010). ${ }^{1}$ In response to these patterns, researchers assert that the best way to promote academic integrity and prevent plagiarism is to strengthen instructorstudent relationships (Bluestein, 2015) and provide opportunities for students to ask questions and seek clarification about ethical source use (Broeckelman-Post, 2008; Buranen, 2009). At the same time, many students feel too intimidated to approach professors with these kinds of questions, fearing that they will expose themselves as incompetent or appear suspicious (Adam et al., 2017; Bluestein, 2015).

In this context, university writing centres can provide an alternative or complementary source of support for students to explore their ideas and concerns in a confidential, non-judgmental space. In many writing centres at Canadian universities, these issues are addressed by student consultants who provide effective and influential peer-to-peer support in individual consultations. These student staff play a key role in providing academic integrity guidance to many student writers.

How student staff perceive their roles in supporting academic integrity is an important but relatively understudied question. A considerable amount of North American research has examined peer-to-peer consultation practices in writing centres; however, there is comparatively little literature focused on how academic integrity is addressed in these consultations. Furthermore, it appears that no Canadian research to date has focused specifically on how student consultants in writing centres address plagiarism and academic integrity concerns in consultations.

To address this gap, the current study uses data gathered through a survey of student staff at a university writing centre to examine the following research questions: How often are plagiarism and academic integrity discussed in writing consultations with student staff, and what kinds of concerns are most common? How confident are student staff in addressing concerns and questions about academic integrity? What do the approaches of student staff suggest about the role of writing centres in supporting academic integrity? By focusing on the perspectives of student consultants at one Canadian institution's writing centre, this chapter will provide deeper insight, not only into current practices, but also into ways that we might further strengthen the role of peer-to-peer learning in supporting the academic integrity of individual students and the institution.

\footnotetext{
${ }^{1}$ I would like to thank the Writing Peers and Writing Support Teaching Assistants at the University of Guelph for participating in the study, and I would like to thank Sarah Elaine Eaton, Julia Christensen Hughes, Clare Bermingham, Sarah Gibbons, Lenore Latta, Kelley Packalen, Jodie Salter, and Ron Ward for their generous and helpful feedback.
} 


\section{Literature Review}

Academic integrity has been well-described in the literature, and there is a growing body of Canadian research on this issue (Eaton, 2017; Eaton \& Edino, 2018; Christensen Hughes \& McCabe, 2006). However, despite the large body of literature, plagiarism, perhaps more so than other types of misconduct, remains difficult to define and address. In the literature review that follows, I briefly describe understandings of plagiarism from the literature and from the University of Guelph specifically before describing the role of writing centres and student staff in preventing plagiarism and supporting academic integrity more broadly.

\section{The Challenge of Defining Plagiarism}

At the University of Guelph, academic misconduct is defined as "behaviour that erodes the basis of mutual trust on which scholarly exchanges commonly rest, undermines the University's exercise of its responsibility to evaluate students' academic achievements, or restricts the University's ability to accomplish its learning objectives" (University of Guelph, 2020b). Plagiarism is considered a subset of academic misconduct and is defined as "misrepresenting the ideas, expression of ideas or work of others as one's own," with examples such as using portions of another work without properly quoting, paraphrasing, or citing; submitting work completed by a third party; or re-submitting work that was completed for another course (University of Guelph 2020b).

Institutional definitions such as the one provided by the University of Guelph make a concerted effort to clarify what plagiarism means, yet the literature demonstrates that a shared understanding of the term and its implications is elusive. Administrators, instructors, and students have a wide variety of understandings of the term (Blum, 2011), and when presented with examples, they often disagree about what constitutes plagiarism and about the seriousness of different types of plagiarism (RobinsonZañartu et al., 2005).

The U.S.-based Writing Program Administrators' best practices document "Defining and Avoiding Plagiarism" argues that a key part of the problem is that definitions are too broad, lumping together deliberate attempts to plagiarize with unintentional citation errors or unskilled source use (Council of Writing Program Administrators, 2003). Because of the ambiguity of many plagiarism definitions and examples, many administrators and instructors have begun to treat plagiarism cases as teachable moments in which to discuss proper source use and integration. A strong proponent of this approach, Rebecca Moore Howard has argued for the "decriminalization" of plagiarism when it is the result of inadequate paraphrasing, a 
practice Howard refers to as "patchwriting" (Howard, 1992, p. 233). ${ }^{2}$ In Howard's view, patchwriting represents a phase in skill development, in which students learn to incorporate the ideas of others into their writing. From this perspective, approaching the topic of plagiarism as an issue of process, practice, and development is a way to open more productive and supportive conversations about academic integrity.

\section{Writing Centres as Spaces for Dialogue and Learning}

Writing centres provide an ideal space for instruction and conversation about source integration and proper citation. In individual consultations, students can discuss their writing at any stage of the process, and most consultants approach this talk with a combination of indirect and direct questions and prompts that can encourage reflection and critical thinking. Though they are sometimes misidentified as "fix-it" shops where students should be sent for grammar correction or to have papers proofread, truly effective writing centres foster dialogue and deep engagement with the writing process. ${ }^{3}$ Importantly, most writing centres in Canadian post-secondary institutions are located outside academic departments and strive to position themselves as neutral ground where students can explore ideas without risk or judgment.

\section{The Role of Student Consultants}

Research has shown that peer-to-peer learning has many advantages, such as promoting metacognition (Stigmar, 2016), supporting cognitive scaffolding (Mackiewicz \& Thompson, 2014), and encouraging motivation and engagement (Topping, 1996). An early proponent of collaborative learning in writing instruction, Kenneth Bruffee (1984) described the key ingredient of peer learning as a conversation between students who are "status equals" (p. 642). In Bruffee's view, there is enormous learning potential in peer-to-peer conversations during the writing process: "[t]he way they talk with each other determines the way they will think and the way they will write" (p. 642). Given these benefits, student consultants have enormous potential to support students in navigating the often-challenging waters of academic

\footnotetext{
${ }^{2}$ In "A Plagiarism Pentimento," Howard defines patchwriting as "copying from a source text and deleting some words, altering grammatical structures, or plugging in one-to-one synonym substitutes" (p. 233).

${ }^{3}$ Stephen North, in the landmark essay "The Idea of a Writing Centre" (1984), asserts that the writing centre is not a place where writers should come (much less be sent to) for grammar correction or writing "first aid." In his view, writing centres are meant to provide the kind of supportive and insightful feedback that is essential to writers' growth, providing a much-needed source of conversation and intervention for writers in process. As he explains, "Writing centers are simply one manifestation — polished and highly visible — of a dialogue about writing that is central to higher education" (p. 440).
} 
integrity; furthermore, writing consultations provide an opportunity for consultants to deepen their own knowledge and understanding of academic integrity concepts.

\section{Writing Centre Approaches to Academic Integrity}

The literature studying the role of writing centres in supporting academic integrity reflects the complicated position that writing centres occupy in relation to plagiarism and academic integrity. A significant portion of the writing centre literature on academic integrity (particularly in the United States) is devoted to confronting critics who misconstrue writing centres' work as unethical, viewing writing help as a form of plagiarism (Clark \& Healy, 1996; Jurecka, 2004; Mackiewicz \& Thompson, 2018). To steer clear of these accusations, most writing centre training guides focus on supporting the writer's learning and development, rather than directing a student about how to perfect a draft (e.g., Gillespie \& Lerner, 2008; Ianetta \& Fitzgerald 2016). As North explains, "[A writing centre's] job is to produce better writers, not better writing" (1984, p. 438). At the same time, many writing centre staff push back on the notion that consultants should be strictly non-directive. As Ianetta and Fitzgerald note, "Alongside the tradition of nondirective tutoring is one that honours modeling writing activities as a way of teaching writers how to eventually do them on their own" (p. 107). Others are even more forceful in their critiques of what they see as institutions chasing untenable notions of individual authorship despite the inherently social nature of writing. As Ginger Jurecka notes, "the concept of an unethical writing center has more to do with archaic notions of ownership within academia than actual moral faltering within the center" $(2004$, p. 2).

Most university and college writing centres, the majority of which are staffed by professionals without faculty status or tenure, must continually balance their commitment to providing a non-judgmental space for students with their responsibility to uphold the policies of the institution. Several writing centres have illustrated this complicated balance by sharing case studies of tutors encountering and addressing suspected plagiarism (Brown et al., 2007; Gruber, 1998; Pelzer, 2019). These experiences of addressing plagiarism can become moments of learning and reflection for tutors and administrators alike, as they wrestle with how to support students while maintaining institutional values. Writing centres still find ways to push boundaries and influence institutional policies, even in this "in-between" space. For example, Brown et al. (2007) describe the activist approach of their student consultant team, which, prompted by a student visit, delved into the research about Turnitin software, and were dismayed by the lack of information being shared with students about how the software works - and its limitations. They decided to share what they learned with students who visited the centre in order to empower students to initiate deeper conversations with their professors about how the use of Turnitin affected their learning in the classroom. A broader aim of this information sharing was to advocate that the university reconsider its use of similarity detection software and other policing strategies for addressing plagiarism. In another approach, writing centre director 
Elizabeth Kleinfeld describes training student consultants to perform "close readings" of students' use of sources using the framework of the Citation Project ${ }^{4}$ (2016, p. 56). By comparing citations to the original source material, student consultants learned how to recognize summarizing, copying, and patchwriting and were able to help writing centre visitors reflect their own source use strategies.

While most writing centres position themselves and their student consultants outside surveillance and policy enforcement, some have taken a more interventionist approach. In a case described by Sibylle Gruber (1998), the writing centre grappled with a student client who blatantly plagiarized and refused to heed a student writing consultant's warnings. The centre ultimately decided to have the student consultant contact the professor, breaking writing centre confidentiality in favour of supporting institutional integrity. As Gruber notes, "we considered ourselves to be in a nowin situation.... We would either fall short of our self-imposed policies and the trust that students put into their interactions with Writing Center staff, or we could be blamed for encouraging and perpetuating unacceptable behavior" (p. 54).

In another example, Pelzer (2019) describes how tutors' uncertainty about how to address possible plagiarism prompted the development of writing centre policies that included a three-strike model and the creation of a plagiarism-monitoring list. The guidelines aimed to guide tutors more clearly about how to "handle, record, and report plagiarism suspicions" (p. 5). Despite this responsibility for surveillance, tutors were also expected to project a non-judgmental stance in consultations and "not to judge a student's authenticity, but rather to educate and provide every resource available to help students improve in this area" (p. 5). In another interventionist approach, Bridgewater, Pounds, and Morley (2019) describe developing a program that required students charged with plagiarism to attend a series of consultations with writing peer tutors. As part of the program, the tutors were required to diagnose the student's plagiarism as intentional or accidental and shape their instruction and advice accordingly. This kind of policy enforcement role might be unpalatable to most writing centres; however, Bridgewater et al. describe it as an opportunity to strengthen the standing and profile of their centre, which "is seen as fulfilling a more academic role than most support centers because it now handles such a challenging issue" (p. 20).

These examples illustrate the complicated and often contradictory situations writing centres and student consultants negotiate in supporting academic integrity on campus. Despite a common desire to provide a judgment-free space for students to talk about plagiarism, writing centres are to varying degrees beholden to the academic integrity priorities and policies of their respective institutions. As Gruber notes, "We need to find the way that justifies our actions to our students, the instructors, the administration, and to ourselves. However, we need to be aware that our position as writing center administrators and writing center staff is a precarious one" (p. 60). In

\footnotetext{
${ }^{4}$ The Citation Project (http://www.citationproject.net/) is described as "a series of research studies on source use. Their purpose is to provide data and analyses that can help with educators' questions about plagiarism, information literacy, and the teaching of source-based writing."
} 
the study that follows, I explore how student consultants approach their work within this complex web of relationships and responsibilities.

\section{Methodology}

In the summer of 2020, participants were recruited for the study from current and recent student writing consultants at the University of Guelph writing centre. ${ }^{5}$ The University of Guelph is a mid-sized comprehensive university, with 29,000 undergraduate and graduate students, including 1,400 international students (University of Guelph, 2020a). The writing centre is located in the university's library and is staffed by 1 manager, 3 full-time professional staff, 10 graduate student writing support teaching assistants (TAs), and 15-20 undergraduate writing peer helpers (Peers). The team provides in-class instruction, specialized workshops and programs, English language support, and a variety of handouts and resources. The centre is best known for its individual writing consultations and provides more than 4,000 sessions (25- or 50-minute consultations) per year to students from all levels and disciplines. Students have the option of attending consultations in person or via an online platform; however, during the COVID-19 pandemic (when data were collected), all consultations were being conducted online.

TAs and Peers complete two days of training each September on conducting effective consultations; supporting writers who have English as an additional language; fostering diversity and inclusivity; writing in the disciplines; making referrals; and using resources. In addition to this, new undergraduate peer helpers receive a full semester of training which addresses similar topics in greater depth. Training of both TAs and Peers emphasizes the importance of students maintaining control over their papers. Consultants are encouraged to avoid writing on student papers other than to highlight areas of focus. Student visits are confidential, and the centre does not report suspected plagiarism, but consultants are encouraged to flag potential plagiarism for students and ensure that students are aware of how their source use may be interpreted by instructors.

To gather the perspectives of the student writing consultants, a 22-question survey was developed and mounted on the Qualtrics platform. The survey included a combination of multiple-choice and open-ended answers. Questions asked about consultants' experiences with supporting student clients with citation, paraphrasing, plagiarism, and academic integrity concerns. Survey results were collected in the Qualtrics platform. Multiple choice answers were analyzed using the report functionality in the Qualtrics software, which produces graphs and tables of responses. Ranking questions were further analyzed using Excel. Text answers were uploaded to NVivo software and analyzed for themes and patterns of language use.

\footnotetext{
5 This study was reviewed by the University of Guelph Research Ethics Board for compliance with federal guidelines for research involving human participants (REB \#20-04-004).
} 


\section{Results and Discussion}

Twenty-two $(N=22)$ responses were gathered from 37 potential respondents over a six-week period, representing a response rate of 57\%. Responses from TAs and Peers were evenly divided between TAs $(n=11)$ and Peers $(n=11)$; however, one TA response was excluded from the data set because the respondent did not answer any of the questions after Question 1 ("Please indicate your most recent role in Writing Services at U of G').

In the section that follows, I describe and interpret the survey results, focusing on key themes that emerge from the data.

\section{Frequency of Topics Related to Plagiarism and Academic Integrity}

One of the basic questions of the study was how often conversations about citing and referencing, paraphrasing, and plagiarism arise in student staff consultations. As shown in Fig. 22.1, conversations about citing and referencing were perceived to be the most common. This finding is not surprising; many students are drawn to our services to "check their references" or to learn how to cite in a particular style. What is interesting, however, is how these conversations can provide openings for student staff to initiate more substantive conversations about citing and referencing. As Peer 5 noted,



Fig. 22.1 Frequency of conversations about citing and referencing, paraphrasing, and plagiarism in consultations 
I used some of [the student's] citation-related queries as a segue into talking about citing and attribution.... The first angle I used was to say that it's important to tell the reader where the information comes from; one example I used was, "if you, [name], told me that A causes B [example from paper], and I want to write that in my paper, I need to write that you said it. And we can do that like this." That opened up the discussion of attribution, and we went from there.

Conversations about plagiarism, at least conversations that explicitly addressed this topic, were less common, with the majority of respondents reporting that it arose in less than $10 \%$ of their consultations. The consultants' comments suggest that rather than discussing plagiarism explicitly, they aim to help students avoid plagiarism by strengthening their skills in related areas, such as citing and paraphrasing. As Peer 11 explained,

It is important not to accuse the student of plagiarizing. If I thought a sentence was not paraphrased adequately (or at all!) then I would ask the student to explain to me what the sentence meant (e.g., "Oh, I am not in science, can you just explain this to me so I understand"). If the student cannot explain the sentence then I would talk about paraphrasing and why it is important for their learning and so that they do not get in trouble (better to hear it from a writing peer, even if a little awkward, than to go through an academic integrity issue with the university).

The sentiments of Peer 11 were widely shared among survey respondents, who studiously avoided even the appearance of challenging or questioning a student's integrity, giving students the benefit of the doubt as much as possible. Shifting the conversation to related skills provided one common way to steer clear of confrontational conversations.

Another aim of the study was to examine more deeply what specific topics are discussed during conversations about citing and referencing, paraphrasing, and plagiarism. To gather this information, the survey asked consultants to rank the most common topics that arose in these discussions (Table 22.1).

Table 22.1 Most common citing and referencing topics reported by consultants (1 being most common)

\begin{tabular}{l|l|l|l}
\hline Rank & Citing \& referencing & Paraphrasing & Plagiarism \\
\hline 1 & Formatting & How to & Fear of accidentally plagiarizing \\
\hline 2 & What to cite & Thoroughness & Defining plagiarism \\
\hline 3 & Placement & Defining & Why instructors care about plagiarism \\
\hline 4 & Style guide & Importance & Contract cheating \\
\hline 5 & Importance of citing & Accuracy & Penalties \\
\hline 6 & Other $^{\text {a }}$ & Other & Other \\
\hline
\end{tabular}

a" Other" text entries: "That/why both in-text citations and a reference list are needed"; "It was fairly common for students to want to discuss the distinctions between footnote/endnote citations and bibliographic citations; usually, the assignment specified which citation style was to be used" b"Other" text entries: "How to avoid accidentally plagiarizing"; "The spirit of referencing" 




Fig. 22.2 Frequency of student and consultant initiation of conversations about academic integrity topics

\section{Initiating Conversations About Academic Integrity}

As Fig. 22.2 illustrates, in consultants' perceptions, initiation of conversations about plagiarism varied depending on specific topic: student clients were more likely than student consultants to begin conversations about citing and referencing, while student consultants were more likely to initiate conversations about paraphrasing or plagiarism. In all topic areas, there were many student consultants who indicated that clients and consultants were equally likely to initiate these conversations.

\section{Student Staff Confidence in Addressing Academic Integrity Concerns}

The data show that confidence in addressing academic integrity concerns was consistently high among the respondents. Across all academic integrity topics (citing and referencing, paraphrasing, and plagiarism), confidence was consistently high, with the majority of respondents agreeing or strongly agreeing that they felt confident having these conversations. On a five-point scale, the average confidence level was 4.7. Moreover, none of the respondents rated their confidence lower than 3 out of 5 . It is reassuring to know that student staff feel comfortable initiating or responding to these issues, and this finding suggests in turn that training can focus on expanding consultants' strategies within these conversations. 


\title{
Strategies for Supporting Academic Integrity
}

Valuable insights were found in consultants' strategies for supporting student skill development in the ethical and effective use of sources. Among the most prominent strategies were those that have been coded as asking curious questions; explaining the value of citing, referencing, and paraphrasing; oral paraphrasing; and using resources.

\section{Curious Questions}

Among the most common strategies cited by the consultants was the use of questions to begin conversations about academic integrity issues. In some cases, this approach dovetailed with the consultant's intention not to appear judgmental, such as in this advice shared by TA 8 :

\begin{abstract}
Remember that plagiarism is a serious offence and accusation, so we want to make sure that we don't accuse someone of something that they've likely done inadvertently. I usually start with, "Where did you get that piece of information? Did you write that?" and have a conversation about the need to give credit where it's due.
\end{abstract}

As this example shows, questions enable consultants to keep students engaged in the consultation and help students think through their choices as writers. They also enable consultants to gather information before deciding what kinds of support are most needed.

\section{Explaining Value}

Another common strategy was to explain the value of citing and referencing, paraphrasing, and avoiding plagiarism $(n=10)$. In this strategy, consultants approached the topic by emphasizing the strategic importance or intrinsic usefulness of the conventions of source use. TA 10 also notes the importance of not promoting what they call "negative views about citing":

I would try to relate to them in that citing can be tedious/annoying, but I would also be conscious about encouraging the negative views about citing. Crediting work is not only important for academic integrity but can also help students find other sources and be more critical about the sources they use.

TA 10's comment demonstrates the in-between place that student staff occupy, empathizing with students' experiences while also coaching students on how to adapt to the conventions and expectations of scholarship. Consistent with this example, student staff consistently asserted the authentic value of academic conventions of source use while at the same time acknowledging the challenge for new students of learning these practices. 


\section{Oral Paraphrasing}

Several student consultants described asking students to explain source ideas out loud, a strategy coded as "oral paraphrasing." Peer 4 explained how they use this approach:

Try to have the student explain to you what the literature is saying in words first without having it in front of them. Many students will paraphrase it well enough without realizing it, and then you can tell them that's all they have to write!

Similarly, TA 4 shared an example of taking a student from source to paraphrase:

Once, I had a student that had trouble understanding how to avoid plagiarism in their paper. The student had a print-out of one of their sources and I asked them to locate the source of the information they were using in their paper. Next, I asked them to verbally explain to me that information. After that, I asked the student to write down what they had just told me. It took a bit of time, but we were able to paraphrase/summarize a small part of the content in a way that didn't incur in plagiarism and the student was able to understand the process of paraphrasing correctly.

These approaches reflect the usefulness of modeling writing strategies for students, as Ianetta and Fitzgerald (2016) note. In her article, "Revisioning InstructorWriting Specialist Collaboration for Paraphrasing Instruction," Silvia Luisa Rossi (2022) describes the importance of providing paraphrasing instruction in a specific writing context. These examples demonstrate how peer-to-peer writing consultations provide an ideal opportunity for this kind of learning.

\section{Using Resources}

The writing centre provides a range of handouts on writing and referencing called "Fast Facts" and guidebooks for consultants and students, and there were many consultant responses $(n=14)$ that described using these as tools in their work with students. In one answer to "What advice would you provide to student consultants about helping students with citation and referencing?" TA 9 suggested:

It's okay to grab a fast facts sheet or pull up the style guide online to help guide you as well.

You can frame it as "Here is a resource that you can use even after the consultation ends;

let's walk through how to find the information you'll need together."

By drawing on resources, student consultants provide another kind of modelling for students, demonstrating that source use and citation requires ongoing learning. These approaches emphasize that knowing how to find answers is as valuable as the answers themselves. 


\section{Additional Training Needs}

As part of the survey, Peers and TAs were asked what additional training might be helpful to them in supporting students with academic integrity. Responses varied, including suggestions to cover referencing styles in greater detail; providing strategies for raising academic integrity concerns in consultations; instruction on how to teach paraphrasing strategies; and incorporating the university's academic integrity policy and website information into training materials.

\section{Cultural Competence}

The most common suggestion $(n=6)$ from student writing consultants was to increase the training about cultural differences in academic writing and how these might lead to unintentional plagiarism. TA 4 explains,

I think that stronger training in cultural differences in academic writing will prove useful.... If the consultant is working with an international student and they find plagiarism in their paper, it's possible that a different writing culture is a reason why the student is (accidentally) plagiarizing. It shouldn't be assumed that they are plagiarizing on purpose!

There were several survey respondents who mentioned the importance of recognizing the additional challenges international students may face in adapting to academic norms in a Canadian academic context. Several noted, as TA 4 does, that international students may come from cultures where plagiarism is understood differently or not discussed at all.

Actively seeking to understand and engage with the diversity of our student population is critical to providing inclusive, welcoming, and accessible services. At the same time, scholars like Arabella Lyon argue that framing academic integrity as culturally rooted can lead to stereotyping international students as inherently "deficient" or "in need of remediation" (2009, p. 224). Similarly, Soni Adhikari (2018) argues that most international students, regardless of cultural background, already do know about plagiarism; problems of citation in their work are more often due to lack of citation skills, difficulties understanding content and context, or challenges with managing time. As Adhikari notes, "Instead of othering students based on how we believe their culture defines plagiarism and intellectual honesty, we can focus on teaching them practical skills that they need to learn" (p. 377). Revisiting our training with this perspective in mind could help our student staff avoid inadvertently othering students who are adapting to new academic and disciplinary conventions.

\section{Role of the Consultant}

An important theme that emerged from the survey responses was student consultants' perceptions of their roles. Consistent in the responses was the notion that consultants 
ought to be supportive insiders who help students learn to understand, negotiate, and apply academic conventions of source use. As Peer 5 explains, "You're helping them to understand the communicative framework they'll be working within for the next four years, and you're essentially providing insider tips!"

Consultants' commitment to being supportive and reassuring was tied to their perceptions of student writers' intent-specifically, consultants by and large interpreted students' lack of citations, patchwriting, or plagiarism as unintentional. Again, Peer 5: "Many students are simply concerned to get it right and not plagiarize accidentally; that's great. In my personal (and therefore anecdotal) experience, those who cheat deliberately are less likely to seek out writing help or guidance."

In consultants' perceptions, the plagiarism they observed was the result of an oversight, lack of knowledge or skill, or cultural differences. These positive beliefs about students' intent coincided, in my view, with consultants' commitment to intervening to protect students from the negative consequences of unintentional plagiarism, such as Peer 11, who noted, "better to hear it from a writing peer, even if a little awkward, than to go through an academic integrity issue with the university."

\section{Conclusion}

This study provides deeper insight into one Canadian writing centre's student consultant experiences of, approaches to, and beliefs about their roles in supporting academic integrity. Consistently throughout the data was the notion that the consultants see themselves as supportive, informed insiders who can intervene at a key point in students' learning and development as academic writers.

From an institutional perspective, these findings suggest that student consultants play important but largely unrecognized roles in supporting academic integrity in our universities. Their ability to relate to and empathize with students makes them an approachable source of information and skill development for anxious students who are concerned about accidentally plagiarizing; similarly, their knowledge and positionality makes them important role models and ambassadors for a wider culture of integrity on campus. By recognizing and promoting the role of these student staff, we may be able to contribute to the broader culture of integrity on our campus.

Key to writing centres' ability to contribute to a culture of integrity is our ability to navigate our complex positioning. Similar to many other university writing centres in Canada and the United States, the University of Guelph's writing centre plays a dual role of supporting students and promoting the values of the institution. Most of the time, these goals are mutually reinforcing; however, academic integrity and plagiarism bring with them a judicial aspect that complicates our role. How do we authentically support students who may be contravening the rules of the institution to which we are accountable? How do we uphold the values of the campus community without alienating individual students who come to us for support? The responses of student writing consultants suggest that they have found a way: by being informative and empathetic guides who are knowledgeable about guidelines and committed to 
understanding students' needs. By explicitly engaging student consultant teams in conversations about academic integrity and plagiarism, we can harness their insights and approaches to communicate our writing centre values to both the institution and our student clients in ways that are clear, respectful, and inclusive.

Two important limitations of this study are its single-institution focus and the small sample size. It is therefore important to understand the findings as informative themes and insights, rather than as predictive of future behaviours. However, future research could include more writing centres to explore the consistency of responses across institutions, allowing us to develop shared approaches to training and resource development. Another important dimension that could be explored in future research is the perspective of student visitors to the writing centre. Gathering these perspectives would help us better understand the experiences and beliefs of students and gauge the fit between consultant and student perspectives. Finally, in light of increasing use of online consultation formats, future research could explore how student consultants approach academic integrity support in a virtual environment.

\section{References}

Adam, L., Anderson, V., \& Spronken-Smith, R. (2017). "It's not fair": Policy discourses and students' understandings of plagiarism in a New Zealand university. Higher Education, 74(1), $17-32$.

Adhikari, S. (2018). Beyond culture: Helping international students avoid plagiarism. Journal of International Students, 8(1), 375-388.

Bluestein, S. A. (2015). Connecting student-faculty interaction to academic dishonesty. Community College Journal of Research and Practice, 39(2), 179-191.

Blum, S. D. (2011). My word!: Plagiarism and college culture. Cornell University Press.

Bridgewater, B., Pounds, E., \& Morley, A. (2019). Designing a writing tutor-led plagiarism intervention program. TLAR, 11-27.

Broeckelman-Post, M. A. (2008). Faculty and student classroom influences on academic dishonesty. IEEE Transactions on Education, 51(2), 206-211.

Brown, R., Fallon, B., Lott, J., Matthews, E., \& Mintie, E. (2007). Taking on Turnitin: Tutors advocating change. Writing Center Journal, 27(1), 7-29.

Bruffee, K. A. (1984). Collaborative learning and the "conversation of mankind." College English, 46(7), 635-652.

Buranen, L. (2009). A safe place: The role of librarians and writing centers in addressing citation practices and plagiarism. Knowledge Quest, 37(3), 24-33.

Christensen Hughes, J. M., \& McCabe, D. (2006). Academic misconduct within higher education in Canada. The Canadian Journal of Higher Education, 36(2), 1-21. http://journals.sfu.ca/cjhe/ index.php/cjhe/article/view/183537/183482

Clark, I. L., \& Healy, D. (1996). Are writing centers ethical? Writing Program Administration, 20(1/2), 32-48.

Council of Writing Program Administrators. (2003). Defining and avoiding plagiarism: The WPA statement on best practices. http://wpacouncil.org

Eaton, S. E. (2017). Comparative analysis of institutional policy definitions of plagiarism: A panCanadian university study. Interchange, 48(3), 271-281. https://doi.org/10.1007/s10780-0179300-7 
Eaton, S. E., \& Edino, R. I. (2018). Strengthening the research agenda of educational integrity in Canada: A review of the research literature and call to action. International Journal for Educational Integrity, 14(1), 5. https://doi.org/10.1007/s40979-018-0028-7

Gillespie, P., \& Lerner, N. (2008). The Longman guide to peer tutoring. Longman.

Gruber, S. (1998). Coming to terms with contradictions: Online materials, plagiarism, and the writing center. The Writing Center Journal, 19(1), 49-71.

Gullifer, J., \& Tyson, G. A. (2010). Exploring university students' perceptions of plagiarism: A focus group study. Studies in Higher Education, 35(4), 463-481.

Howard, R. M. (1992). A plagiarism pentimento. Journal of Teaching Writing, 11(2), 233-245.

Ianetta, M., \& Fitzgerald, L. (2016). The Oxford guide for writing tutors: Practice and research.

Jurecka, G. (2004). Intellectual property paranoia and the writing center. Praxis: A Writing Center Journal, 2(1), 1-4.

Kleinfeld, E. (2016). Using citation analysis in writing center tutorials to encourage deeper engagement with sources. Praxis: A Writing Center Journal, 13(2), 52-58.

Lyon, A. (2009). "You Fail": Plagiarism, the ownership of writing, and transnational conflicts. College Composition and Communication, 61(2), W222-W239.

Mackiewicz, J., \& Thompson, I. (2014). Instruction, cognitive scaffolding, and motivational scaffolding in writing center tutoring. Composition Studies, 42(1), 54-78.

Mackiewicz, J., \& Thompson, I. (2018). Spoken written-language in writing center talk. Linguistics and Education, 47, 47-58.

North, S. M. (1984). The idea of a writing center. College English, 46(5), 433. https://doi.org/10. 2307/377047

Pelzer, E. (2019). Writing center tutors take on plagiarism. Praxis, 16(3), 2-5.

Robinson-Zañartu, C., Peña, E. D., Cook-Morales, V., Peña, A. M., Afshani, R., \& Nguyen, L. (2005). Academic crime and punishment: Faculty members' perceptions of and responses to plagiarism. School Psychology Quarterly, 20(3), 318-337.

Rossi, S. L. (2022). Revisioning instructor-writing specialist collaboration for paraphrasing instruction. Academic Integrity in Canada. Springer.

Stigmar, M. (2016). Peer-to-peer teaching in higher education: A critical literature review. Mentoring \& Tutoring: Partnership in Learning, 24(2), 124-136.

Topping, K. J. (1996). The effectiveness of peer tutoring in further and higher education: A typology and review of the literature on JSTOR. Higher Education, 32(3), 321-345.

University of Guelph. (2020a). About. University of Guelph Website. https://www.uoguelph.ca/ about

University of Guelph. (2020b). Academic Misconduct-VIII. Undergraduate Degree Regulations and Procedures. Undergraduate Calendar. https://www.uoguelph.ca/registrar/calendars

Kim Garwood has worked in academic support at the University of Guelph since 2007 and has collaborated on a national research project on academic integrity. She has a $\mathrm{PhD}$ from the University of Waterloo. 
Open Access This chapter is licensed under the terms of the Creative Commons Attribution 4.0 International License (http://creativecommons.org/licenses/by/4.0/), which permits use, sharing, adaptation, distribution and reproduction in any medium or format, as long as you give appropriate credit to the original author(s) and the source, provide a link to the Creative Commons license and indicate if changes were made.

The images or other third party material in this chapter are included in the chapter's Creative Commons license, unless indicated otherwise in a credit line to the material. If material is not included in the chapter's Creative Commons license and your intended use is not permitted by statutory regulation or exceeds the permitted use, you will need to obtain permission directly from the copyright holder.

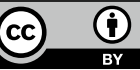

